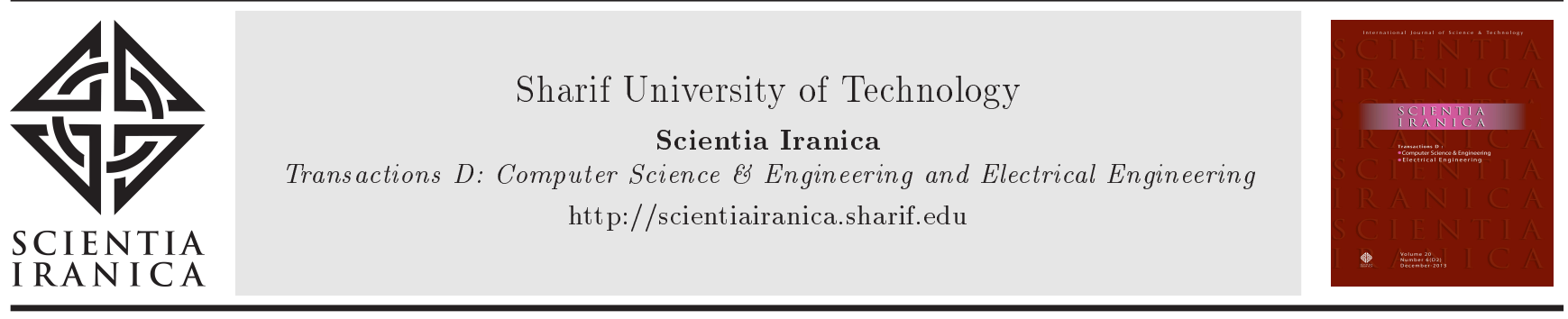

\title{
A methodology for examining the chaotic behavior of CoP signal during quiet standing based on empirical mode decomposition method
}

\author{
R. Hajipour ${ }^{\mathrm{a}}$, F. Asadollahzadeh Shamkhal ${ }^{\mathrm{b}}$, and H.R. Kobravi ${ }^{\mathrm{a}, *}$ \\ a. Research Center of Biomedical Engineering, Mashhad Branch, Islamic Azad University, Mashhad, Iran. \\ b. Department of Electrical Engineering, Faculty of Engineering, Ferdowsi University of Mashhad, Iran.
}

Received 9 February 2019; received in revised form 28 June 2019; accepted 26 August 2019

\author{
KEYWORDS \\ Quiet standing; \\ Center of Pressure \\ $(\mathrm{CoP})$; \\ Empirical Mode \\ Decomposition \\ (EMD); \\ Chaotic dynamics; \\ Recurrence \\ Quantitative Analysis \\ (RQA).
}

\begin{abstract}
A key parameter for analyzing the human balance dynamics in the standing position is the Center of Pressure (CoP). However, no conclusive idea has been posed with respect to elicited dynamics of the $\mathrm{CoP}$ signal in quiet standing so far. In this paper, a heuristic algorithm was proposed to prove the chaotic behavior of the CoP signal with high confidence. In the proposed algorithm, first, the deterministic and non-deterministic (either stochastic or chaotic) components of the CoP signal were extracted using the Empirical Mode Decomposition (EMD) method. Then, the nonlinear features of the extracted components such as fractal dimension, Lyapunov exponent, correlation dimension, and alpha parameter were computed. Then, based on the quantitative value of the computed features, the chaotic component was selected from the extracted components. Finally, through the Recurrence Quantitative Analysis (RQA), the selected chaotic component was reanalyzed to ensure the correct selection of the chaotic component. In this respect, the kind of CoP dynamics can be determined with high confidence. The analyzed CoP signals were recorded through some experiments on 12 healthy subjects aging 20 to 70 years old. The results of this study indicated that $\mathrm{CoP}$ was a chaotic signal with high confidence.

(C) 2021 Sharif University of Technology. All rights reserved.
\end{abstract}

\section{Introduction}

The position of the ground reaction force called the Center of Pressure (CoP) plays a key role in controlling the balance during standing [1-4]. CoP, by itself, can be a criterion for measuring the stability during quiet standing [1]. Not only may the magnitude of the $\mathrm{CoP}$ but its direction of displacement, or heading, of the $\mathrm{CoP}$ can provide further insight into

*. Corresponding author. Tel.: +985136629467 E-mail addresses: hajipourr91@gmail.com (R. Hajipour); Fateme_A_shamkhal@yahoo.com (F. Asadollahzadeh Shamkhal); Hkobravi@mshdiau.ac.ir (H.R. Kobravi)

doi: $10.24200 /$ sci.2019.52901.2943 the $\mathrm{CoP}$ [2]. In other words, the $\mathrm{CoP}$ measurement is usually performed as an indicator for maintaining balance and postural control. Hence, in recent years, many researchers have investigated the behavior of CoP signal as a nonlinear signal [5-19]. The eminent conducted studies on this issue have focused on two main axes: (a) study of the complicated nature of the CoP signal and process of standing balance [6-10] and (b) the changes of the CoP features under different conditions [11-19]. In the first group of studies, researchers have investigated whether the process of standing is chaotic. Some of these studies have failed to definitely determine whether the behavior of the CoP signal was chaotic or stochastic [10]. For instance, Collins and Luca considered the behavior of the CoP signal for the first time and suggested that this behav- 
ior had a sign of random correlated noise [7]. Later, other researchers have suggested that the complex and unpredictable behavior of the motor sensor control system can be indicative of the chaos in controlling the status of the individual's body $[8,9]$. Ghomashchi et al. could not reach a definite conclusion about the chaotic or stochastic behavior of the CoP signal [10]. In the second group of studies, in order to analyze the changes in the nonlinear features of the $\mathrm{CoP}$ in different conditions, researchers have employed different methods. For instance, Kuznetsov et al. and Gurses and Celik employed fractal analysis and Correlation Dimension (CD) estimation of CoP signal to investigate the process of standing balance and body sway, respectively $[13,14]$. However, a controversial question has remained without a definite answer, i.e., Is the CoP a chaotic signal or not? The Langevin equation was also used for modeling the CoP dynamics $[15,16]$. They analyzed the change of CoP dynamics, excluding the visual feedback, age, and disease severity $[15,16]$. Although the change in the balance dynamics can be determined through this approach, nothing can be claimed about the nature of the $\mathrm{CoP}$ during standing. In addition, the original Langevin equation describes a specific stochastic process called Brownian motion [17], while no conclusive evidence has been presented so far for specifying the Brownian nature of the CoP signal. Snoussi et al. studied the behavior of CoP by decomposing the signal into its components using the Empirical Mode Decomposition (EMD) method [18]. They emphasized that the presence of deterministic and stochastic components accompanied by chaotic components in the CoP signal might result in mixing up the chaotic behavior of CoP with a stochastic behavior. Therefore, they used the EMD method to extract the chaotic component of the CoP signal based on the analysis of the Lyapunov Exponent (LE). Through the EMD, a time series is partitioned into Intrinsic Mode Functions (IMF) without leaving the time domain. Such modes may provide a better understanding of different signals in the data. In other words, EMD can facilitate extracting the time components and showing different behavioral aspects of the nonlinear and nonstationary signals, hence being useful for this study. However, the analysis based on the LE alone may yield some misleading results due to the presence of either discretization errors or measurement noise. Therefore, in this study, an analysis algorithm was proposed based on the EMD method to select the chaotic component among the extracted components with high confidence.

\section{Methods and materials}

\subsection{Data collection}

The experiments were conducted on 12 healthy individuals with no history of disease including six males and

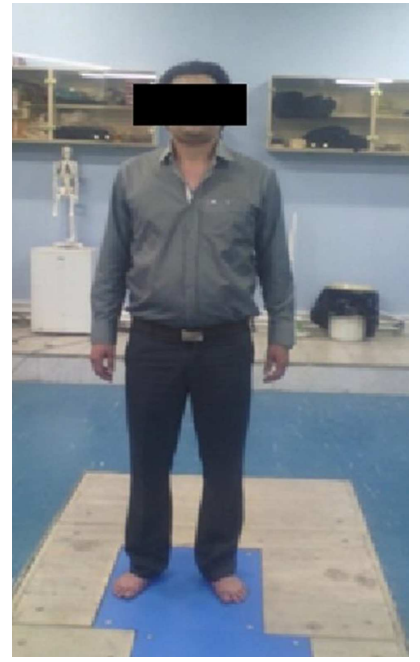

Figure 1. The healthy subject standing quietly on a force plate.

six females between 20 and 70 years who participated in the experiments. Although the age range is wide, according to the received medical consultations, the balance quality in the individuals over 50 was not significantly affected due to aging. Therefore, there was no need to categorize the individuals according to their age. The individuals were asked to stand quietly with barefoot on the Force Plate (9286A, KISTLER) and eyes open during experiments. Each trial lasted two minutes and the sampling frequency was $100 \mathrm{~Hz}$. Individuals maintained their upright posture while CoP signal was recorded during each experiment. Figure 1 shows the experimental setup.

\subsection{The proposed analysis methodology}

Figure 2 shows the flowchart of the proposed analysis methodology. In this suggested algorithm, first, the CoP data were decomposed into their components using a nonlinear preprocessing method called EMD. Then, Fractal Dimension (FD) was computed for the extracted components. Since the possibility of computational errors may be raised owing to data discretization, a fractional number instead of an integer number can be obtained. Since there is no trivial approach to reducing the quantization error, a heuristically simple approach was selected. In this study, Higuchi method was used for computing the fractal dimension [19]. According to this approach, the fractal dimension is computed through the following exponential relation [19]:

$$
\langle L(k)\rangle \propto k^{-D},
$$

where $D$ is the fractal dimension, $k$ the length of the time interval, and $\langle L(k)\rangle$ the average value of the lengths associated with the time series constructed by the raw data [19]. In this study, the computed fractal dimension $(D)$ was about 1 . Therefore, $1 \pm 0.01$ 


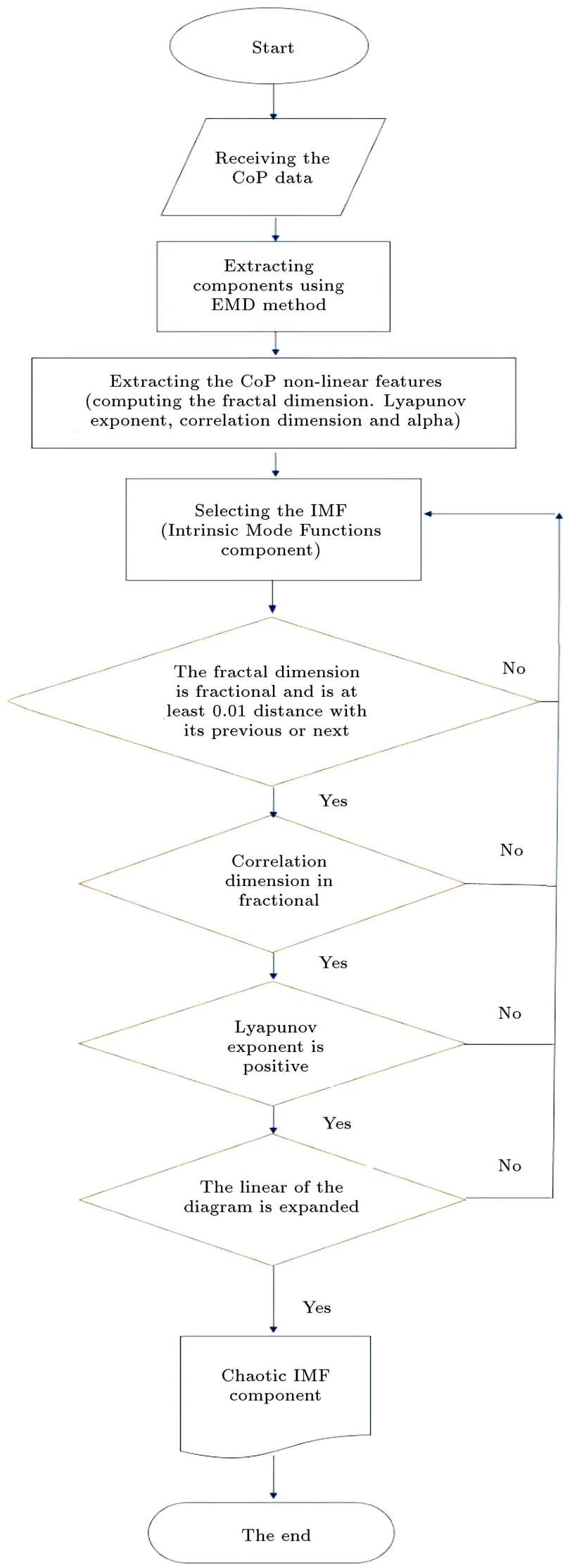

Figure 2. The flowchart of the Center of Pressure (CoP) signal chaotic component selection algorithm. (or $1 \pm$ less than 0.01 ) does not significantly change the $L$ value, since 0.01 (or less) can be negligible in comparison with 1 . However, it can also be misleading because the $L$ value will not be as outlier data as that one can recognize. Therefore, in this study, 0.01 was regarded as a computational margin. In this regard, those components whose fractal dimension were fractional and were at least 0.01 more or less than the integer value were selected as the candidate chaotic components. Next, CD and LE were computed for the candidate components [20]. The selected components should contain fractional CD and positive LE. Finally, through another nonlinear analysis method called Detrended Fluctuation Analysis (DFA), logarithmic diagrams related to the extracted components were plotted. The component containing the logarithmic diagram with a sufficiently expanded linear area and the least misleading possibility of estimating the scaling exponent was calculated as the slope of a straight line fit to the log-log graph. Therefore, the component whose logarithmic diagram had the most expanded linear area was selected as the component, which is a chaotic component with high confidence.

\subsubsection{EMD analysis}

EMD is an experimental method for analyzing the natural signals that are usually non-linear and nonstationary [5,21]. It was first introduced by $\mathrm{Wu}$ and Huang in [21]. EMD decomposed a nonlinear nonstationary signal into a finite number of oscillatory functions called IMF. IMFs of a signal were then extracted in a process called the screening process, which is implemented through the following stages:

1. Specifying all the local extrema of the signal.

2. Obtaining the upper and lower envelope of the signal.

3. Calculating the mean of the upper and lower envelopes of the signal:

$$
e(t)=\frac{X_{L}(t)+X_{U}(t)}{2}
$$

4. Subtracting the envelope mean from the entering signal:

$$
d(t)=X(t)-e(t)
$$

The screening process continued for the remaining signal $d(t)$ until reaching the final specified conditions. The extracted component will be IMF if it meets the following two conditions:

1. Throughout the dataset, the number of extremes and crossing the zero should be equal or maximally have one unit of difference; 
2. In each point, the envelope mean defined by the local maximums and the envelope defined by the local minimums should be zero. In other words, an IMF should be a symmetric function at about zero.

If the above conditions are met, the extracted component will be considered as IMF. Otherwise, the screening process continues on the remaining signal until extracting the first IMF.

The screening process can be halted by any of the following predefined conditions:

1. When the remaining component is so small that it is smaller than a predefined significant amount;

2. When the remaining component changes into a monotonic function from which no other IMF can be extracted.

Upon the end of the screening process, the mentioned nonlinear analysis was carried out.

\subsubsection{DFA analysis}

DFA is a known approach to quantification of the complexity of non-stationary signals based on the analysis of short-term and long-term autocorrelation of a signal. DFA algorithm output is a parameter called $a$ which is the slope of the logarithmic graph. It indicates the statistical self-affinity of the signal. In this method, the signal squares least distance from the signal trend is analyzed as a function of the scale parameter [22-24]. For $X(i)$, DFA algorithm was applied, as shown in the following:

1. Calculation of the value of the $X(i)$ oscillation:

$$
y(k)=\sum_{i=1}^{k}\left(X(i)-X_{\text {avg }}\right)^{y} .
$$

2. Calculation of the average value of the signal oscillation relative to its trend:

$$
F(n)=\sqrt{\frac{1}{N}} \sum_{k=1}^{N}\left(y(k)-y_{n}(k)\right)^{2} .
$$

3. Plotting $\log F(n)$ logarithmic graph to $\log n$ :

$$
F(n) \approx n^{a} .
$$

In the plotted logarithmic graph, the slope of the linear region is referred to as parameter $a$. In fact, $a$ is the slope of a region where $\log F$ has a linear relationship with $\log n$.

\subsubsection{Calculating the LE}

Since no differential equation model could describe the raw data or IMFs, the LE should be computed with the available signals. Therefore, the Wolf wellknown method was applied [25]. According to the Wolf method, the LE is defined by the long-term evolution of nearby orbits in the reconstructed m-dimensional phase state and it can be computed through Eq. (7) [25]:

$$
\lambda_{1}=\frac{1}{t_{M}-t_{0}} \sum_{k=1}^{M} \log _{2} \frac{L^{\prime}\left(t_{k}\right)}{L\left(t_{k-1}\right)},
$$

where $\lambda_{1}$ is the LE, $L\left(t_{k-1}\right)$ the Euclidian distance between a data point located at the nearest neighborhood of a point in the phase portrait related to a time instance $\left(t_{k-1}\right), L^{\prime}\left(t_{k}\right)$ the Euclidian distance between the next replaced data point and the next point in the phase portrait related to a time instance $\left(t_{k}\right)$, and $M$ is the total number of the replacement steps; further, $t_{0}$ and $t_{M}$ represent the initial and the last time instances.

\subsubsection{Analysis of IMF5 using RQA}

In the last step of the proposed algorithm, the complexity of the selected IMF and raw CoP signal was compared to obtain the desired results. In other words, the selected IMF is expected to be a more complex signal than the raw CoP signal. Therefore, it can be concluded that the selected IMF is the chaotic component of the $\mathrm{CoP}$ signal with high confidence. In line with the objectives of this study, Recurrence Quantitative Analysis (RQA) was employed. RQA is a nonlinear method for analyzing dynamic systems, especially for measuring the complexity of a signal [26]. This method was established to quantify Recursive Plots (RP) on the basis of short-scale structures [27]. It makes an RP in which some variables that measure different aspects of the dynamics of COP data are extracted [28]. Generally, RPs are a graphic expression of the trajectories of dynamics of system mode space $[26,27,29]$. A relatively simple method widely used in some previous researches, RQA is characterized by the main principle, i.e., the phase space of a single time series can only be reconstructed through time delay [30]. The output of the RQA is a set of features for quantitative analysis of recursive graphs. In this study, a feature called determinism (DET) was employed. This measure is related to the predictability of the system dynamics. The DET is defined as the percentage of the recurrence points of diagonal lines to the total number of recurrence points. It can be measured as follows:

$$
D E T=\frac{\sum_{l=l_{\min }}^{N} l P(l)}{\sum_{i, j=1}^{N} R(i, j)},
$$

where $P(l)$ is the distribution of the diagonal lines and $l$ is the number of points forming the line. This measure is related to the predictability of the system dynamics. 


\section{Results}

\subsection{Analysis of the IMFs}

Figure 3 shows an example of $\mathrm{CoP}$ decomposition into 11 IMF components using the EMD algorithm. At the next step, the fractal dimension of the obtained
IMFs is calculated. At this stage, the components with fractional dimensions and a value of at least 0.01 more or less than an integer value were selected as the candidate chaotic components. The calculated values for the selected IMFs for two sample participants are given in Tables 1 and 2. According to these tables,
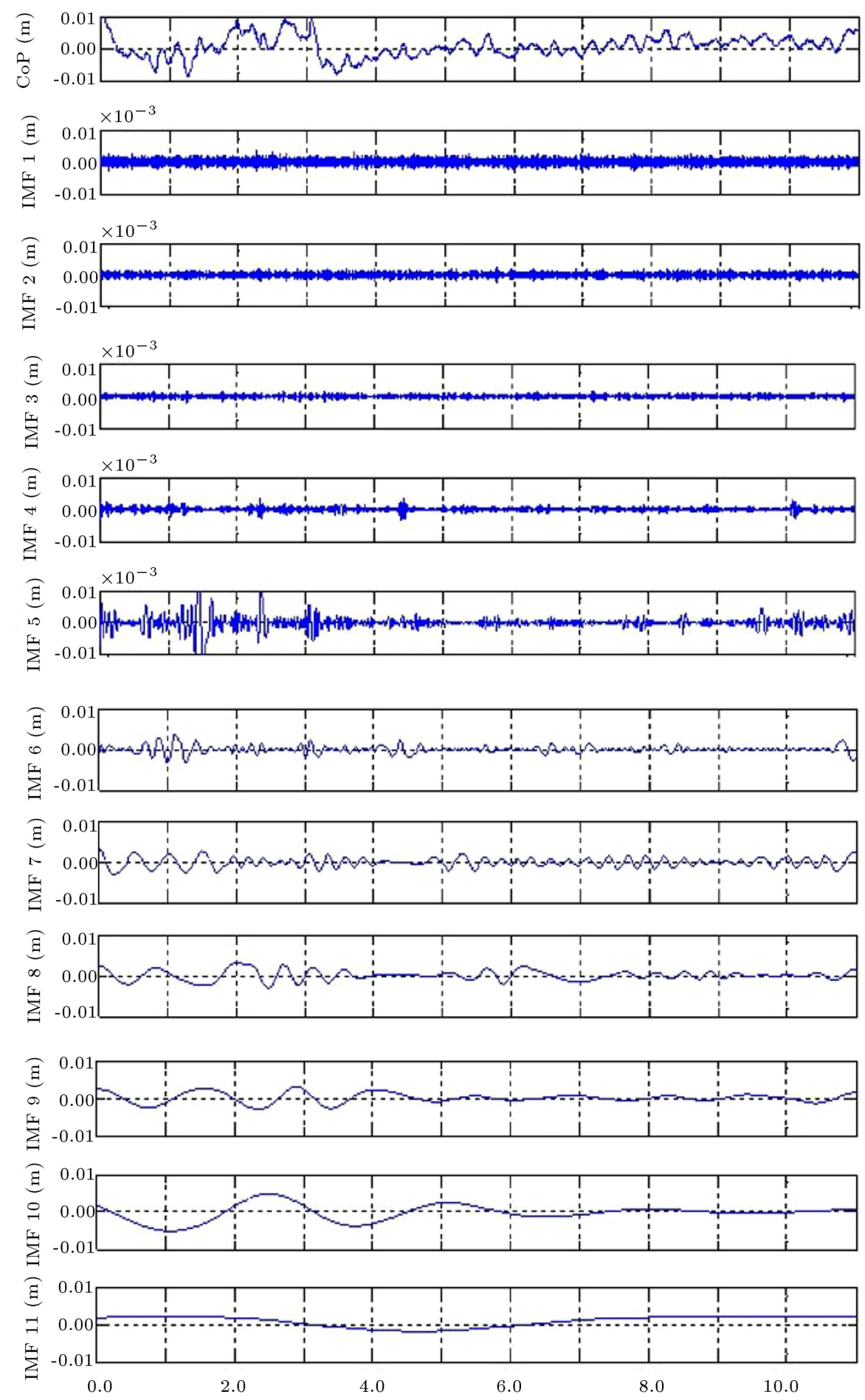

Figure 3. Center of Pressure (CoP) signal taken from a healthy subject during quiet standing for 2 minutes and extracted Intrinsic Mode Functions (IMFs) from raw Center of Pressure (CoP) signal using the Empirical Mode Decomposition (EMD) algorithm. 
Table 1. Parameters of fractal dimension, correlation dimension, Lyapunov exponent, and parameter $\alpha$ related to raw Center of Pressure (CoP) signal and its extracted Intrinsic Mode Functions (IMFs) (related to Subject 1).

\begin{tabular}{cccccccccccccc}
\hline & \multicolumn{10}{c}{ Signal } \\
\cline { 2 - 21 } & CoP & IMF1 & IMF2 & IMF3 & IMF4 & IMF5 & IMF6 & IMF7 & IMF8 & IMF9 & IMF10 & IMF11 & IMF12 \\
\hline FD & 1.64 & 2.00 & $\mathbf{1 . 9 6}$ & $\mathbf{1 . 6 7}$ & $\mathbf{1 . 1 8}$ & $\mathbf{1 . 0 2}$ & 1.00 & 1.00 & 1.00 & 0.99 & 0.99 & 1.00 & 0.99 \\
CD & 1.57 & 1.69 & $\mathbf{1 . 6 0}$ & $\mathbf{1 . 4 5}$ & $\mathbf{1 . 3 9}$ & $\mathbf{1 . 3 6}$ & 1.66 & 1.75 & 1.60 & 1.79 & 1.69 & 1.64 & 1.76 \\
LE & 0.56 & 8.85 & $\mathbf{8 . 7 2}$ & $\mathbf{8 . 3 0}$ & $\mathbf{7 . 4 3}$ & $\mathbf{6 . 5 1}$ & 5.71 & 5.19 & 4.40 & 3.60 & 2.85 & 2.32 & -0.00 \\
$\boldsymbol{\alpha}$ (DFA) & 1.04 & 0.41 & $\mathbf{1 . 4 4}$ & $\mathbf{2 . 0 3}$ & $\mathbf{2 . 2 4}$ & $\mathbf{2 . 3 0}$ & 2.30 & 2.31 & 2.31 & 2.31 & 2.30 & 2.31 & 2.31 \\
\hline
\end{tabular}

Table 2. Parameters of fractal dimension, correlation dimension, Lyapunov exponent, and parameter $\alpha$ related to raw Center of Pressure (CoP) signal and its extracted Intrinsic Mode Functions (IMFs) (related to Subject 2).

\begin{tabular}{|c|c|c|c|c|c|c|c|c|c|c|c|c|c|}
\hline \multirow[b]{2}{*}{ Feature } & \multicolumn{13}{|c|}{ Signal } \\
\hline & CoP & IMF1 & IMF2 & IMF3 & IMF 4 & IMF5 & IMF6 & IMF 7 & IMF 8 & IMF9 & IMF 10 & IMF11 & IMF 12 \\
\hline FD & 1.78 & 2.00 & 1.95 & 1.69 & 1.20 & 1.04 & 1.01 & 1.00 & 1.00 & 0.99 & 0.99 & 0.99 & 0.99 \\
\hline CD & 1.62 & 1.64 & 1.64 & 1.58 & 1.52 & 1.24 & 1.40 & 1.55 & 1.68 & 1.81 & 1.80 & 1.72 & 1.73 \\
\hline LE & 0.69 & 8.65 & 8.70 & 8.25 & 7.56 & 6.79 & 5.32 & 4.83 & 4.33 & 3.59 & 3.05 & 2.91 & -0.00 \\
\hline$\alpha($ DFA $)$ & 0.89 & 0.46 & 1.53 & 2.07 & 2.25 & 2.29 & 2.30 & 2.31 & 2.31 & 2.31 & 2.31 & 2.31 & 2.31 \\
\hline
\end{tabular}

Components $2-5$ satisfied the mentioned conditions. Of note, there were some significant similarities among the computed fractal dimensions despite the individuals' considerable weight, height, and age differences. This finding was achieved based on the calculated mean and standard deviations of the computed fractal dimensions among the participants. The computed standard deviation was measured as $1.02 \pm 0.01$, which was quite smaller than the computed mean. These similarities were also indicative of some similar movement patterns during standing among the healthy individuals. At the next step, to verify the accuracy of the chaotic nature of the selected components, the CD and LE of the selected IMFs were calculated. According to Tables 1 and 2, the calculated CD for Components 2-5 is also a fractional number, and LE of Components 2-5 are large and positive. These results confirmed the chaotic behavior of the selected component and the effect of the measurement noise and computational error on the results was almost negligible. Of note, the calculated LE related to the second-to-fifth components were greater than those related to the raw signal. According to what was mentioned above, analysis of the raw CoP signal can be misleading due to the stochastic and deterministic components of $\mathrm{CoP}$ signal. In addition, similar results among all participants were obtained. Tables 1 and 2 present the obtained results for the twosample subjects, despite the similar results among the participants.

\subsection{Selection of an IMF using DFA}

Finally, the components 2-5 were analyzed using DFA. Computing the $\alpha$ value is the output of DFA. The $\alpha$ value is the slope of a line fitted to the linear region of the logarithmic graph (Eq. (6)). Thus, existence

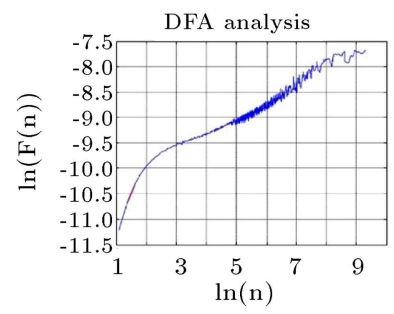

(a)

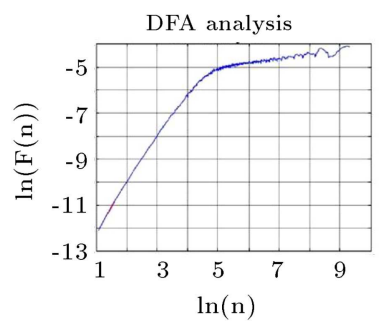

(c)

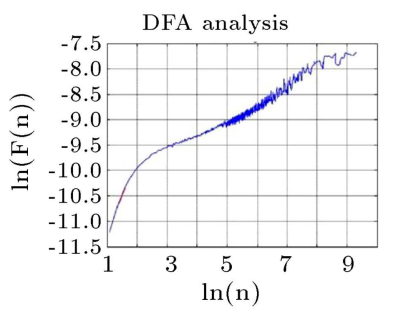

(b)

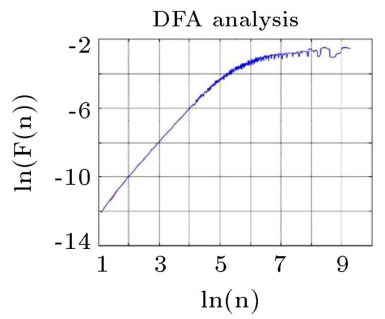

(d)

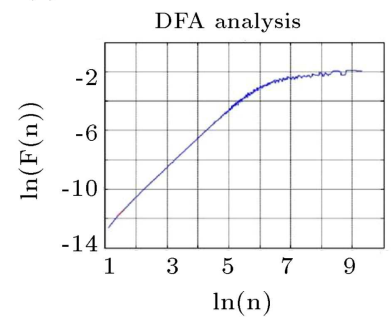

(e)

Figure 4. logarithmic graph of Detrended Fluctuation Analysis (DFA) analysis related to raw Center of Pressure (CoP) signal (a) and logarithmic graph of IMF2 to IMF5 (b-e).

of calculation error is inevitable. Nevertheless, as the range of the linear region becomes wider the calculation error will be reduced more. According to Figure 4, among Components 2-5, Component 5 has a 
wider linear area. Therefore, IMF5 was selected as a component providing more precise information about the chaotic nature of the $\mathrm{CoP}$ signal. Apparently, the analysis of the complexity of the balancing process via IMF5 is more suitable.

The calculated parameter $\alpha$ related to IMF5 for all individuals was substantially larger than 0.5. Hence, it can be claimed that the extracted IMF5 signal for all individuals was an anti-persistent, signal which is indicative of the emergent behaviors in the balancing process.

\subsection{Analysis of the Selected IMF Using RQA}

As mentioned earlier, at the last step of the proposed algorithm, the complexity of the selected IMF and raw $\mathrm{CoP}$ signal was compared to elaborate the obtained results. This comparison was made using a quantitative feature (DET) extracted by RQA. Table 3 shows the computed DET values as well as the related mean and the standard deviation values. Accordingly, it can be concluded that the calculated DET related to the extracted IMF5 component $(0.98 \pm 0.01)$ had a larger value than the corresponding raw CoP signal $(0.89 \pm 0.06)$, thus proving that the nature of the IMF5 component was more complex than that of the raw CoP signal.

The finding that the calculated standard deviation was small is indicative of the closeness of the calculated DET values for all the individuals. This finding is interestingly consistent with the previously mentioned conclusion about the presence of a similar movement pattern during the balance process in the able-bodied individuals.

\section{Discussion}

\subsection{Groups}

This study analyzed the obtained data on healthy subjects of different ages. The main focus was put on the healthy subjects to identify the nature of $\mathrm{CoP}$, while the able-bodied subjects were quietly standing. The CoP signal can be considered as a criterion for quantitative analysis of balance quality in the quiet standing position. Therefore, identifying the nature of the specified CoP signal during the quiet standing can be an informative approach to human dynamic balance analysis. Analysis of the balance dynamics through quantitative criteria can be useful in diagnosing some balance disorders and quantifying balance loss among patients suffering from balance disorders during upright standing. In addition, designing an adequate control strategy for motor rehabilitation needs acquiring deep knowledge about the characteristics of balance dynamics during quiet standing. According to the obtained results, it can be argued with high confidence that the balance dynamics in the able-bodied subjects is chaotic.

\subsection{Dynamic similarity}

Some evidence in recent years has proved the similarities among the characteristics of balance dynamics for different individuals [31]. Consequently, an insignificant gap can be observed among the computed nonlinear qualitative features of the signal such as CoP, caused by body sway during quiet standing, for different healthy subjects. The calculated mean and standard deviation of the computed fractal dimensions among the participants were $1.02 \pm 0.01$. The quite smaller value of the calculated standard deviation than the calculated mean showed significant similarities between the computed fractal dimensions of the extracted IMFs despite the considerable differences between the subjects' weight and height, thus confirming this hypothesis. In addition, IMF5 was selected as a component capable of providing more precise information about the chaotic nature of the CoP signal among all participants. The parameter $\alpha$ calculated by DFA and related to IMF5 in all subjects was substantially larger than 0.5 , indicating that the extracted IMF5 signal was an anti-correlated one in all subjects. More interestingly, the complexity of IMF5 was analyzed using RQA method, the results of which demonstrated that the calculated values of DETs for all subjects were close. The calculated mean and standard deviations of the computed DET among the participants were $0.98 \pm 0.01$. All these results not only are consistent with the evidence proving the presence of similar movement patterns in the balancing process in the able-bodied subjects but also point to the presence of a specific nonlinear dynamic balance during the standing position which is chaotic, according to our interpretations.

\subsection{Chaotic nature of CoP and IMF5}

Application of the EMD method to decomposing the CoP signal to local oscillations that make the nonstationary signal has been previously investigated [19]. These components can bear valuable information about

Table 3. Determinism (DET) values of raw Center of Pressure (CoP) signal and IMF5 component in all subjects.

\begin{tabular}{ccccccccccccccc}
\hline & \multicolumn{10}{c}{ Subject } \\
\cline { 2 - 12 } Signal & IMF1 & IMF2 & IMF3 & IMF4 & IMF5 & IMF6 & IMF7 & IMF8 & IMF9 & IMF10 & IMF11 & IMF12 & Mean \pm STD \\
\hline Raw CoP & 0.95 & 0.78 & 0.91 & 0.92 & 0.78 & 0.93 & 0.82 & 0.89 & 0.89 & 0.93 & 0.97 & 0.94 & $0.89 \pm 0.06$ \\
IMF5 & 0.99 & 0.98 & 0.99 & 0.99 & 0.95 & 0.99 & 0.97 & 0.99 & 0.99 & 0.99 & 0.99 & 0.99 & $0.98 \pm 0.01$ \\
\hline
\end{tabular}


the nature of the signal; however, analysis results obtained from some computed nonlinear features may be tricky, especially when the features such as LE and fractal dimensions should be computed. Measurement noise and possible computational errors may undermine the accuracy of the computational results. In our proposed multistage algorithm, the result related to each stage ratifies the archived result related to the last stages. The positive LE along with the fractional fractal and $\mathrm{CD}$ of $\mathrm{CoP}$ related to all subjects may prove the chaotic nature of CoP [32]. Nevertheless, the results elucidate that the calculated LE related to the second to fifth components are greater than the calculated LE related to the raw CoP signal. It can be shown that the analysis of the raw CoP signal can be misleading. In addition, at the end of the algorithm, IMF5 was selected as the chaotic component of the CoP bearing the most precise information about the CoP dynamic. The calculated DET measure, in all subjects, related to extracted IMF5 component had a larger value than the corresponding raw CoP, proving that the IMF5 component was of more complex nature than the raw CoP signal. This result also ratifies that analysis of the raw $\mathrm{CoP}$ signal can be misleading. According to the results, we believe that the CoP signal contains the chaotic local oscillations and consequently has a chaotic nature. Nevertheless, to analyze the balance dynamics and its variations during the upright standing, scrutinizing the extracted IMF5 is more appropriate.

\subsection{Comparison with the other methods}

The previous works mostly extracted the nonlinear features of raw CoP signal to analyze the CoP dynamics [5-19]. However, we believe that the presence of deterministic and stochastic components accompanied by chaotic components in CoP signal may result in mixing up the different behaviors of $\mathrm{CoP}$ with each other. Therefore, the analyses based on feature extraction from the raw CoP can be misleading. Thus, this study applied the EMD method to analyze the CoP signals. It is worth noting that the EMD method has been used so far to analyze the CoP [5], but the only aim here was to distinguish between eyes-open and eyes-closed conditions [5]. Some other researchers only analyzed the changes of $\mathrm{CoP}$ dynamics with respect to age and disease severity $[12,16]$, or sought to show the relationship between a nonlinear feature and actual balance ability [6]. Overall, none of the previous works [6-10] have posed clear and confident claims with respect to the $\mathrm{CoP}$ signal dynamics. By using the algorithm proposed in our study, a clear and confident claim has been posed about CoP dynamics. According to the presented study, it can be asserted that CoP signal is a chaotic signal.

\section{Conclusion}

Determining the nature of Center of Pressure (CoP) signal is significantly challenging. In this study, a new algorithm was proposed to determine the nature of $\mathrm{CoP}$ based on some known nonlinear analyses. Although the authors of this study believed that non-chaotic components of the CoP signal would lead to wrong analysis of CoP, how to choose a suitable decomposition approach was still in debate. An appropriate decomposition approach should be able to detect the obscured dynamic properties of the signal. Therefore, a conventional decomposition method based on the orthogonal functions cannot be of help because the extracted components should contain some information about the nature of $\mathrm{CoP}$. In this regard, inspired by the results of the recent research, this study employed the Empirical Mode Decomposition (EMD) method to decompose the CoP signal. However, the effects of the measurement noise and computational errors always undermine the accuracy of the computational results from evaluating the nonlinear features commonly used for identifying the chaotic nature of signals. Therefore, in this study, a multistage algorithm was utilized. At each stage, some nonlinear features were computed. Then, some criteria at each stage were employed, and some extracted components were selected as the candidate chaotic components. Finally, the fifth extracted component was recognized as the chaotic component of the CoP signal with high confidence. Afterward, Recurrence Quantitative Analysis (RQA) was employed to analyze the complexity of the fifth extracted component. The results of this analysis were in agreement with the previously obtained result. Therefore, it was concluded that $\mathrm{CoP}$ could be recognized as a chaotic signal with high confidence.

Of note, despite the considerable differences among the individuals' weights and heights, some significant similarities were observed among the calculated quantitative features. These similarities suggested that there might be a similar movement pattern during quiet standing which could be consistent with the fundamental concept of muscle synergy.

\section{Acknowledgement}

This work was supported by the Biomedical Research Center at Islamic Azad University of Mashhad. The authors declare that there is no conflict of interests.

\section{References}

1. Popović, M.R., Pappas, I.P.I., Nakazawa, K., et al. "Stability criterion for controlling standing in ablebodied subjects", J. Biomech., 33(11), pp. 1359-1368 (2000). 
2. Rhea, C.K., Kiefer, A.W., Haran, F.J., et al. "A new measure of the CoP trajectory in postural sway: dynamics of heading change", Med. Eng. Phys., 36(11), pp. 1473-1479 (2014).

3. Caballero, C., Barbado, D., and Moreno, F.J. "What COP and kinematic parameters better characterize postural control in standing balance tasks?", J. Mot. Behav., 47(6), pp. 550-562 (2015).

4. Tan, A.M., Fuss, F.K., Weizman, Y., and Azari, M.F. "Centre of pressure detection and analysis with a highresolution and low-cost smart insole", Procedia Eng., 112, pp. 146-151 (2015).

5. Pachori, R.B., Hewson, D.J., Snoussi, H., et al. "Analysis of center of pressure signals using empirical mode decomposition and Fourier-bessel expansion", 2008 IEEE Region 10 Conference', TENCON 2008, pp. 1-6 (2008).

6. Liu, K., Wang, H., Xiao, J., et al. "Analysis of human standing balance by largest lyapunov exponent", Comput. Intell. Neurosci., 2015, p. 158478 (2015).

7. Collins, J.J. and Luca, C.J.D. "Open-loop and closedloop control of posture: A random-walk analysis of center-of-pressure trajectories", Exp. Brain Res., 95(2), pp. 308-318 (1993).

8. Pascolo, P.B., Marini, A., Carniel, R., et al. "Posture as a chaotic system and an application to the Parkinson's disease", Chaos Solitons Fractals, 24(5), pp. 1343-1346 (2005).

9. Ladislao, L. and Fioretti, S. "Nonlinear analysis of posturographic data", Med. Biol. Eng. Comput., 45(7), pp. 679-688 (2007).

10. Ghomashchi, H., Esteki, A., Sprott, J.C., et al. "Identification of dynamic patterns of body sway during quiet standing: Is IT a nonlinear process?", J Bifurc. Chaos, 20, pp. 1269-1278 (2010).

11. Blaszczyk, J.W. and Klonowski, W. "Postural stability and fractal dynamics", Acta Neurobiol. Exp. (Warsz.), 61(2), pp. 105-112 (2001).

12. Doyle, T.L.A., Dugan, E.L., Humphries, B., et al. "Discriminating between elderly and young using a fractal dimension analysis of centre of pressure", Int. J. Med. Sci., 1(1), pp. 11-20 (2004).

13. Kuznetsov, N., Bonnette, S., Gao, J., et al. "Adaptive fractal analysis reveals limits to fractal scaling in center of pressure trajectories", Ann. Biomed. Eng., 41(8), pp. 1646-1660 (2013).

14. Gurses, S. and Celik, H. "Correlation dimension estimates of human postural sway", Hum. Mov. Sci., 32(1), pp. 48-64 (2013).

15. Bosek, M., Grzegorzewski, B., and Kowalczyk, A. "Two-dimensional Langevin approach to the human stabilogram", Hum Mov Sci, 22(6), pp. 649-660 (2004).
16. Bosek, M., Grzegorzewski, B., Kowalczyk, A., et al. "Degradation of postural control system as a consequence of Parkinson's disease and ageing", Neurosci. Lett., 376(3), pp. 215-220 (2005).

17. Lemons, D.S. and Gythiel, A. "Paul Langevin's 1908 paper 'On the Theory of Brownian Motion' ['Sur la théorie du mouvement Brownien,' C. R. Acad. Sci. (Paris) 146, 530-533 (1908)]", American Journal of Physics, 65(11), pp. 1079-1081 (1997).

18. Snoussi, H., Hewson, D., and Duchene, J. "Nonlinear chaotic component extraction for postural stability analysis", Conf. Proc. Annu. Int. Conf. IEEE Eng. Med. Biol. Soc. IEEE Eng. Med. Biol. Soc. Annu. Conf., 2009, pp. 31-34 (2009).

19. Torre, F.C.-D. la, González-Trejo, J.I., Real-Ramirez, C.A., et al. "Fractal dimension algorithms and their application to time series associated with natural phenomena", J. Phys.: Conf. Ser., 475, pp. 1-10 (2013).

20. Ding, M., Grebogi, C., Ott, E., Sauer, T., et al. "Estimating correlation dimension from a chaotic time series: when does plateau onset occur?", Phys. Nonlinear Phenom., 69(3), pp. 404-424 (1993).

21. Wu, Z. and Huang, N.E. "Ensemble empirical mode decomposition: a noise-assisted data analysis method", Adv. Adapt. Data Anal., 1(1), pp. 1-41 (2009).

22. Golińska, A.K. "Detrended fluctuation analysis (DFA) in biomedical signal processing: Selected examples", Stud. Log. Gramm. Rhetor., 29, pp. 107-115 (2012).

23. Minamisawa, T., Takakura, K., and Yamaguchi, T. "Detrended fluctuation analysis of temporal variation of the center of pressure (COP) during quiet standing in parkinsonian patients", J. Phys. Ther. Sci., 21(3), pp. 287-292 (2009).

24. Bardet, J.M. and Kammoun, I. "Asymptotic properties of the detrended fluctuation analysis of long-rangedependent processes", IEEE Trans. Inf. Theory, 54(5), pp. 2041-2052 (2008).

25. Wolf, A., Swift, J.B., Swinney, H.L., et al. "Determining Lyapunov exponent from a time series", Physica., 16D, pp. 285-317 (1984).

26. Zbilut, J.P. and Webber, C.L. "Recurrence quantification analysis: introduction and historical context", Int. J. Bifurc. Chaos, 17(10), pp. 3477-3481 (2007).

27. Iwaniec, J., Klepka, A., and Uhl, T. "Recurrence plots and RQA analysis for damage detection in mechanical systems", in 'Proceedings of the 8th International Conference on Structural Dynamics' EURODYN, 2011, pp. 2476-2482 (2011).

28. Negahban, H., Sanjari, M.A., Karimi, M., et al. "Complexity and variability of the center of pressure time series during quiet standing in patients with 
knee osteoarthritis", Clin. Biomech., 32, pp. 280-285 (2016).

29. Masia, M., Bastianoni, S., and Rustici, M. "Recurrence quantification analysis of spatio-temporal chaotic transient in a closed unstirred Belousov-Zhabotinsky reaction", Phys. Chem. Chem. Phys., 3(24), pp. 55165520 (2001).

30. Apthorp, D., Nagle, F., and Palmisano, S. "Chaos in balance: Non-linear measures of postural control predict individual variations in visual illusions of Motion", PLOS ONE, 9(12), p. e113897 (2014).

31. Torres-Oviedo, G. and Ting, L.H. "Muscle synergies characterizing human postural responses", J. Neurophysiol., 98(4), pp. 2144-2156 (2007).

32. Hosseini, S.A.A. "Chaos and bifurcation in nonlinear inextensional rotating shafts", Sci. Iran., 26(2), pp. 856-868 (2019).

\section{Biographies}

Rahel Hajipour was born in 1983 in Mashhad, Iran. She received her BSc (2005) degree in Biomedical Engineering from Isfahan University and MSc (2014) degree in Biomedical Engineering from Islamic Azad University of Mashhad, Iran. Her main research interest is nonlinear analysis of human motions. Currently, she is a Teacher Assistant at Islamic Azad University of Mashhad and a medical devices expert at Mashhad
University of Medical Science.

Fateme Asadollahzadeh Shamkhal received the BS degree in Biomedical Engineering (Bioelectric) from Islamic Azad University of Mashhad, 2015, and the MS degree in Biomedical Engineering (Bioelectric) from Ferdowsi University of Mashhad, Iran, 2018. Her research interests concern the biomedical signal processing aimed to develop the new diagnosis methods and rehabilitation technologies.

Hamid Reza Kobravi received the BS degree in Electrical Engineering from Ferdowsi University, Mashhad, 2000 as well as the MS and $\mathrm{PhD}$ degrees in Biomedical Engineering from Iran University of Science and Technology, Tehran, 2004 and 2011, respectively. From 2001 to 2011, in cooperation with Neural Technology Center in Iran University of Science and Technology, he worked on the design and development of a variety of neural prostheses applicable to movement restoration in the patients with spinal cord injury. At present, he is an Assistant Professor in Biomedical Engineering at Azad University of Mashhad (Iran). He is the Head of Neuromuscular System Control Lab. in Azad University of Mashhad. His research interests concern using the artificial intelligence in controlling and modeling of the biological systems, biomedical signal processing, and control of complex systems. 